\title{
BALM: Watching the Formation of Tethered Bilayer Lipid Membranes with submicron lateral resolution
}

\author{
J. BOMPARD ${ }^{1}$, O. MANITI ${ }^{1}$, R. ABOUKHACHFE ${ }^{2}$, D. AUSSERRE ${ }^{3 *}$, A. GIRARD-EGROT ${ }^{1 *}$
}

Affiliations:

${ }^{1}$ Univ Lyon, Université Claude Bernard Lyon 1, CNRS, Institut de Chimie et Biochimie Moléculaires et Supramoléculaires, ICBMS, UMR 5246, GEMBAS Team, Lederer building, 1 rue Victor Grignard, F-69622 Villeurbanne, France

${ }^{2}$ Lebanese university, Faculty of technology, Hisbe Street, Saida, Lebanon

${ }^{3}$ Institut des Matériaux et Molécules du Mans, IMMM UMR CNRS 6283, Le Mans Université, Avenue Olivier Messiaen, 72085 Le Mans, France.

*: Co-corresponding authors agnes.girard-egrot@univ-lyon1.fr and Dominique.Ausserre@univ$\underline{\text { lemans.fr }}$

\section{Supplements}

\section{Figure S1:}

Figure 1A curves reproduced with POPC liposomes.

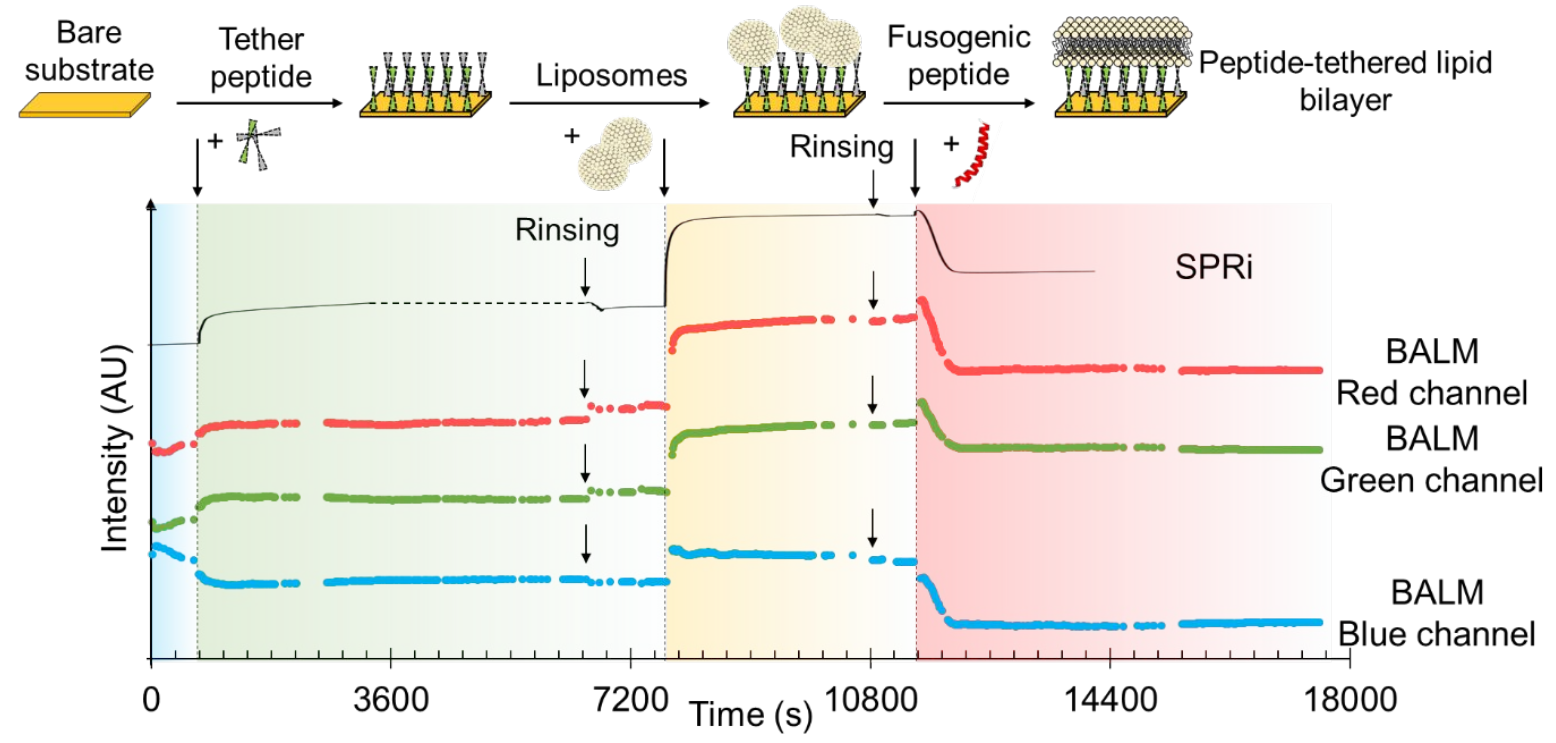

The BALM curves show a good qualitative correspondence with the SPR curve, as we can easily follow every step of the tBLM formation process. With the help of the cartoon on top of the figure, we can identify the tether peptides grating, the subsequent liposome anchoring, and their fusion in a flat lipid bilayer. 


\section{Figure S2:}

Characteristic times of the fusion stage with POPC liposomes.

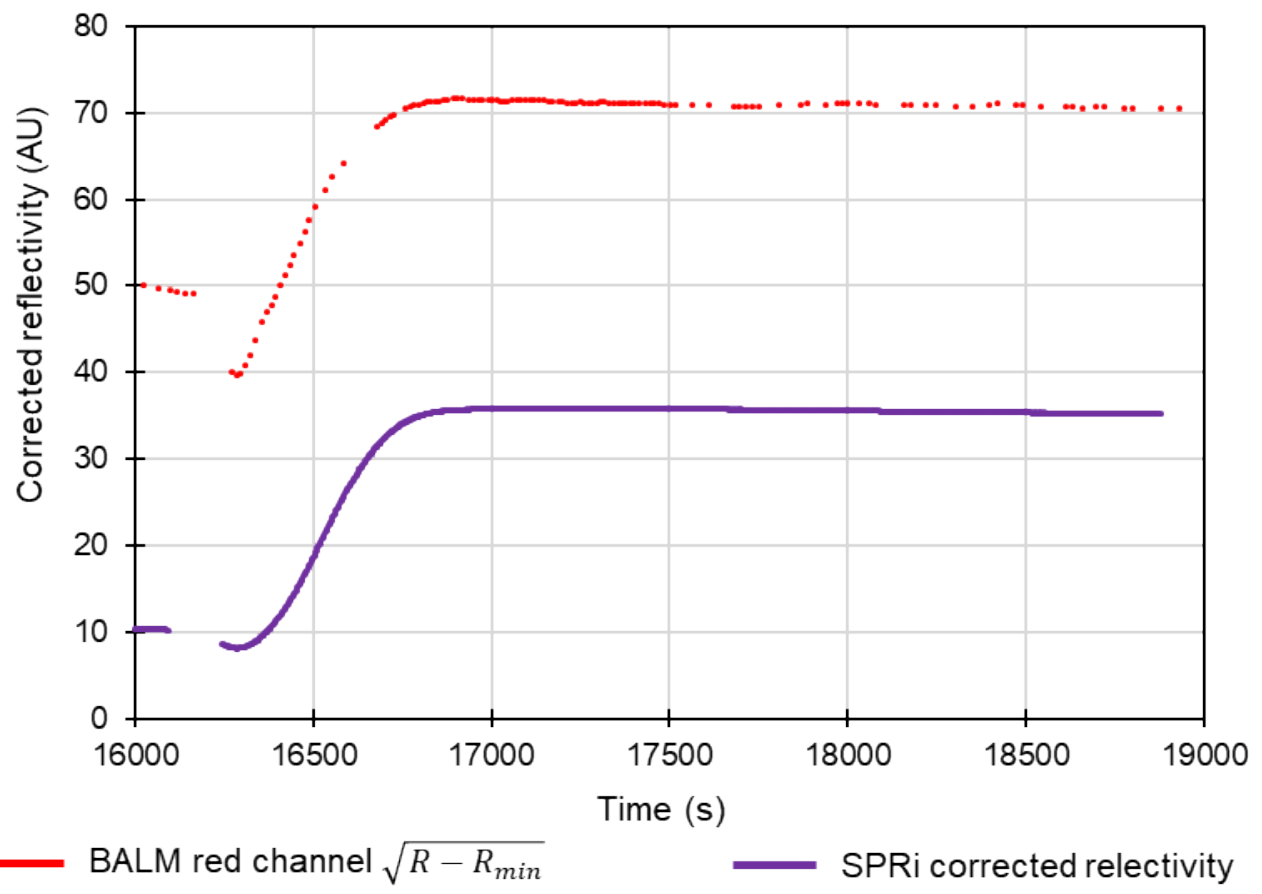

Evolution of the BALM and SPR (corrected) reflectivities during the fusion stage. By contrast with the biomimetic system, the characteristic time of the kinetics as seen with BALM $\sqrt{R-R_{\min }}$ and SPR is the same. Hence, the two techniques can be used indifferently in affinity constant measurements. We note a more sigmoidal shape with SPR, more exponential with BALM. We attribute this difference to a weaker sensitivity of SPR to distant bilayers (the upper part of the liposomes). The SPR reflectivity $x$ has been redistributed according to $y=a x+b$, with $a=-1.5, b=70$, with the value of $|a|$ of order 1 showing comparable sensitivity. 


\section{Figure S3:}

\section{The limits of SPR linearity with thickness.}

When treating the electromagnetic field profile in the evanescent wave as a constant between $z=0$ and $z=\Lambda$, the average variation of the refractive index is $\Delta\langle n\rangle=\left(n_{1}-n_{0}\right)\langle\phi\rangle$, with $\langle\phi\rangle=\frac{1}{\Lambda} \int_{0}^{\Lambda} \phi(z) d z$

The thickness of the layer with refractive index $n_{1}$ is $e$, hence $\langle\phi\rangle=\frac{1}{\Lambda} \int_{0}^{\mathrm{e}} 1 d z=\frac{e}{\Lambda}$ and

$$
\Delta\langle n\rangle=\left(n_{1}-n_{0}\right) \frac{e}{\Lambda}
$$

This is what we used in the main text, Equation (1).

When taking into account the field profile $E(z)=e^{-z / \Lambda}$ in the evanescent wave, we must replace in $\Delta$ $\langle n\rangle$ the simple average $\langle\phi\rangle=\frac{1}{\Lambda} \int_{0}^{\Lambda} \phi(z) d z$ by the weighted average $\langle E \phi\rangle=\frac{1}{\Lambda} \int_{0}^{\infty} e^{-z / \Lambda} \phi(z) d z$, with $\phi$ $(z)=1$ for $0<z \leq e$ and $\phi(z)=0$ elsewhere. It gives:

$$
\langle E \phi\rangle=\frac{1}{\Lambda} \int_{0}^{\mathrm{e}} e^{-z / \Lambda} d z=\left(1-e^{-\frac{e}{\Lambda}}\right)
$$

The variation of the average refractive index is therefore:

$$
\Delta_{\Lambda}\langle n\rangle=\left(n_{1}-n_{0}\right)\left(1-e^{-\frac{e}{\Lambda}}\right)
$$

, with the index $\Lambda$ referring to the account of the field profile. This is what we used in the main text, Equation (2). Comparison of Equation (S2-1) and Equation (S2-2) makes a simple distortion factor appear:

$$
\Delta_{\Lambda}\langle n\rangle / \Delta\langle n\rangle=\frac{\left(1-e^{-x}\right)}{x}
$$

, with $=\frac{e}{\Lambda}$.

The distortion factor is plotted in the opposite figure. Note that the relative error made ( $=1-$ distortion factor $)$ is

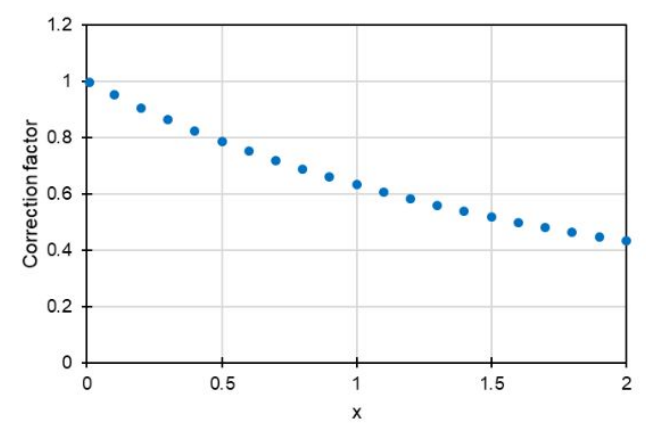
already as much as $10 \%$ with a $\Lambda / 5$ layer thickness, for instance a $20 \mathrm{~nm}$ layer if $\Lambda=100 \mathrm{~nm}$ as for the SPRi apparatus used in this study. It reaches $37 \%$ for $100 \mathrm{~nm}$ liposomes treated as homogeneous objects (i.e. with constant index profile). It follows that the liposome thickness (or surface excess) is underestimated by SPR by $37 \%$. Knowing that the equivalent lipid layer thickness per footprint is $6 \alpha h$, the distorted SPR signal will correspond to $3.81 \alpha h$ and must be interpreted as such. When exploiting the data, the correction factor will be (1/distortion factor).

As an additional remark, we want to stress out a difficulty with the definition of $\Lambda$. We took it as the decay length of the electromagnetic field because the refractive index affects the field propagation equations, including their imaginary form. Yet, it is sometimes interpreted as the decay length of the electromagnetic energy, probably because the measured reflectivity is an intensity. We believe it is not correct for SPR because the field to energy conversion takes place outside the evanescent wave. By contrast, it might be correct for instance when expressing a TIRF intensity (Total Internal Reflection Fluorescence) because the energy conversion is inside. Therefore, the effective penetration depth for SPR and TIRF could differ by a factor of 2 . 
Figure S4:

The principle of the BALM measurement.

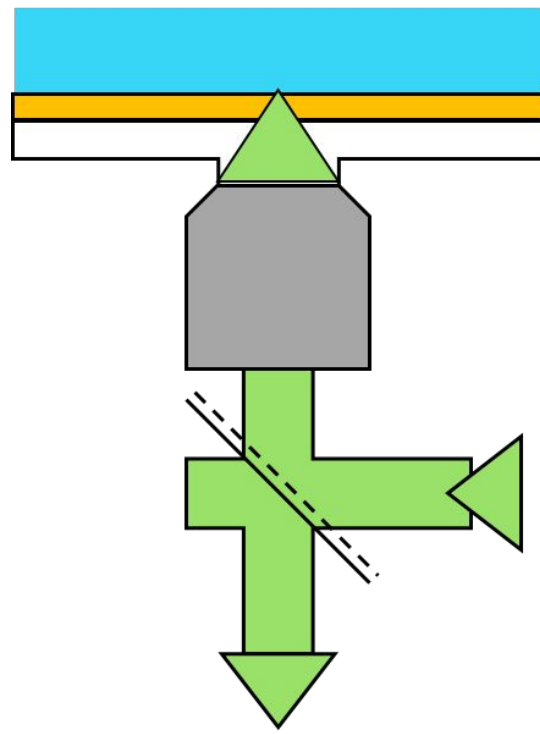

Light out, surface reflectivity
Added adsorbed layer

\section{Water cell}

ARA layer window

Objective lens

Light in

The figure on the left schematizes the BALM setup. The objective lens is linked to the front (lower) side glass window by a drop of immersion oil. Thus, the sole reflecting element is the top (back) surface of the window. Moreover, the presence of the ARA layer with an optimal thickness makes this reflection very weak. When a thin layer is added on that layer, the reflectivity changes to higher (positive contrast) or lower (negative contrast) values, but as long as the added layer stays counted in nanometers, it remains very weak. The low reflectivity results from multiple interferences in the ARA layer (bare substrate) or in the superimposed ARA and added layers (sample). 


\section{Figure S5:}

Quantitative comparisons.

The stage by stage adjustment of the $R_{\min }$ values was conducted by independently adjusting the red and green $R_{\min }$ values. This was made possible by optimizing the linearity between the two BALM $(R)$ $=\sqrt{\frac{R-R_{\min }}{R_{0}-R_{\min }}}$. Since the denominator is constant over a stage, it is equivalent and sufficient to optimize the linearity between the two numerators $n(R)=\sqrt{R-R_{\min }}$. The 4 curves below show the variation of $n(R)$ Green as a function of $n(R)$ Red obtained after each two parameters optimization in each step.
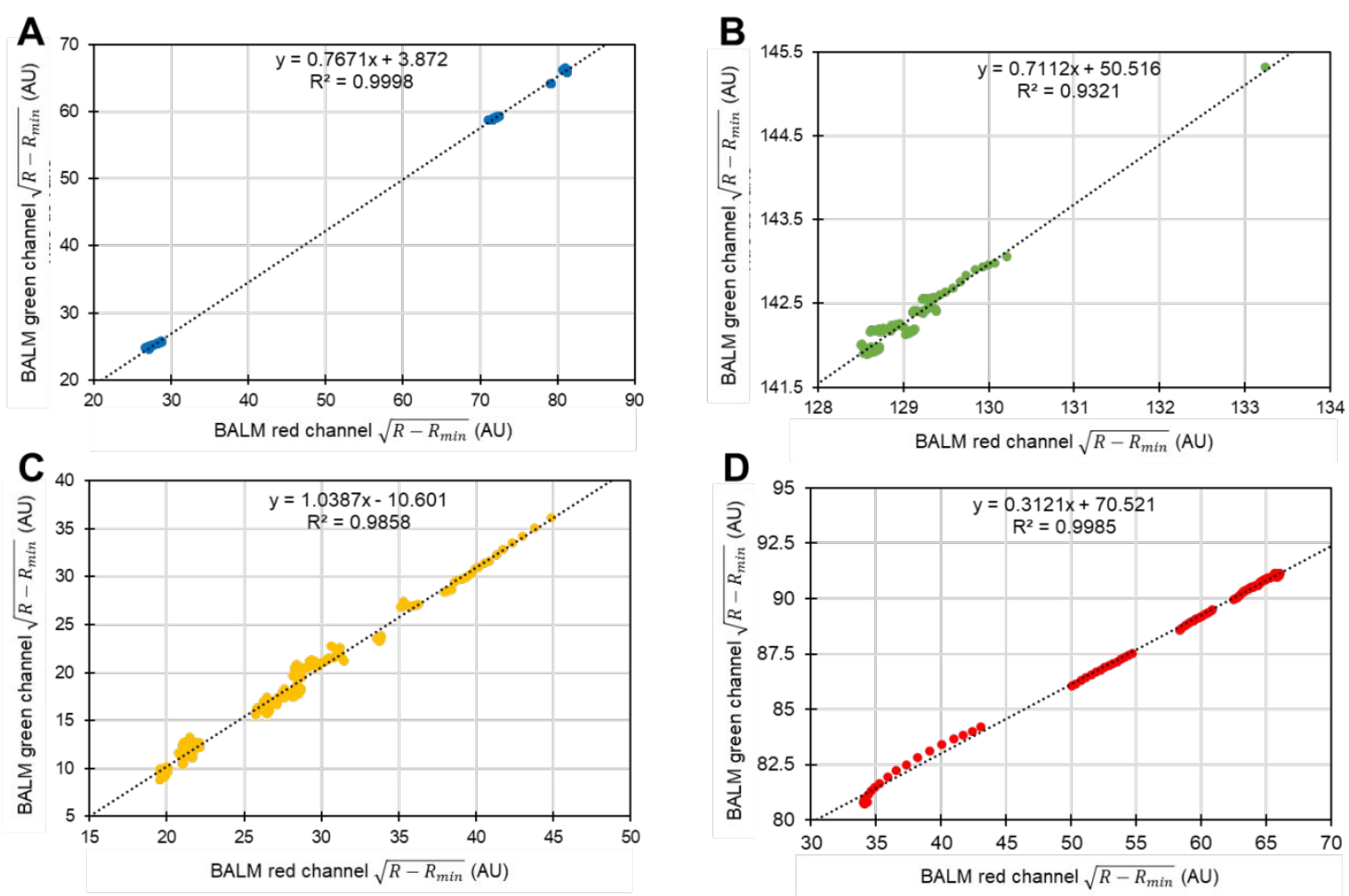

A: Buffer + rinsing stages. B: Tether grafting stage. C: Liposomes anchoring stage. D: fusion stage.

Once the $R_{\min }$ parameters fixed, the linearity between BALM and SPR was checked by adjusting $a$ and $b$ in

$$
f(R)=a * \Delta R(S P R)+b
$$

in such a way that the saturation levels are the same. The first member (Red and Green) and second member of this formula after adjustment are plotted in the figure below. The saturation levels are marked with the blue lines. 


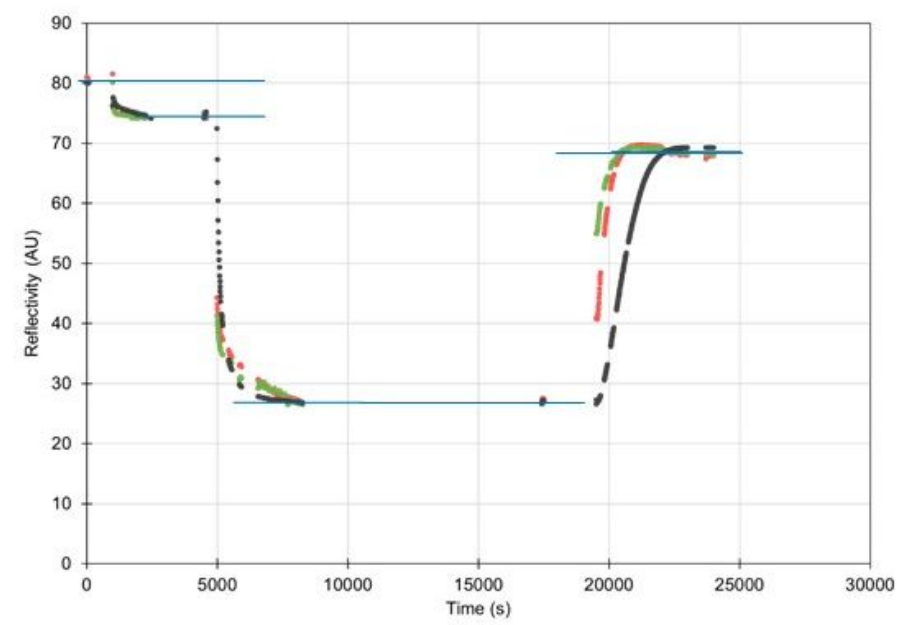

We explain the differences between the SPR and BALM curves by different sensitivities to the distant lipids forming the liposomes. A difference is also observed in the apparent kinetic constants, but this one is specific to the precise experiment that we reported, i.e. not related to the optical techniques, see Supplement 6.

To relativize the importance of these differences, the next figure shows the dispersion of the raw SPRi and BALM measurements in the 16 SPRi spots (A) and in 10 zoomed subzones drawn in the BALM images (B). 

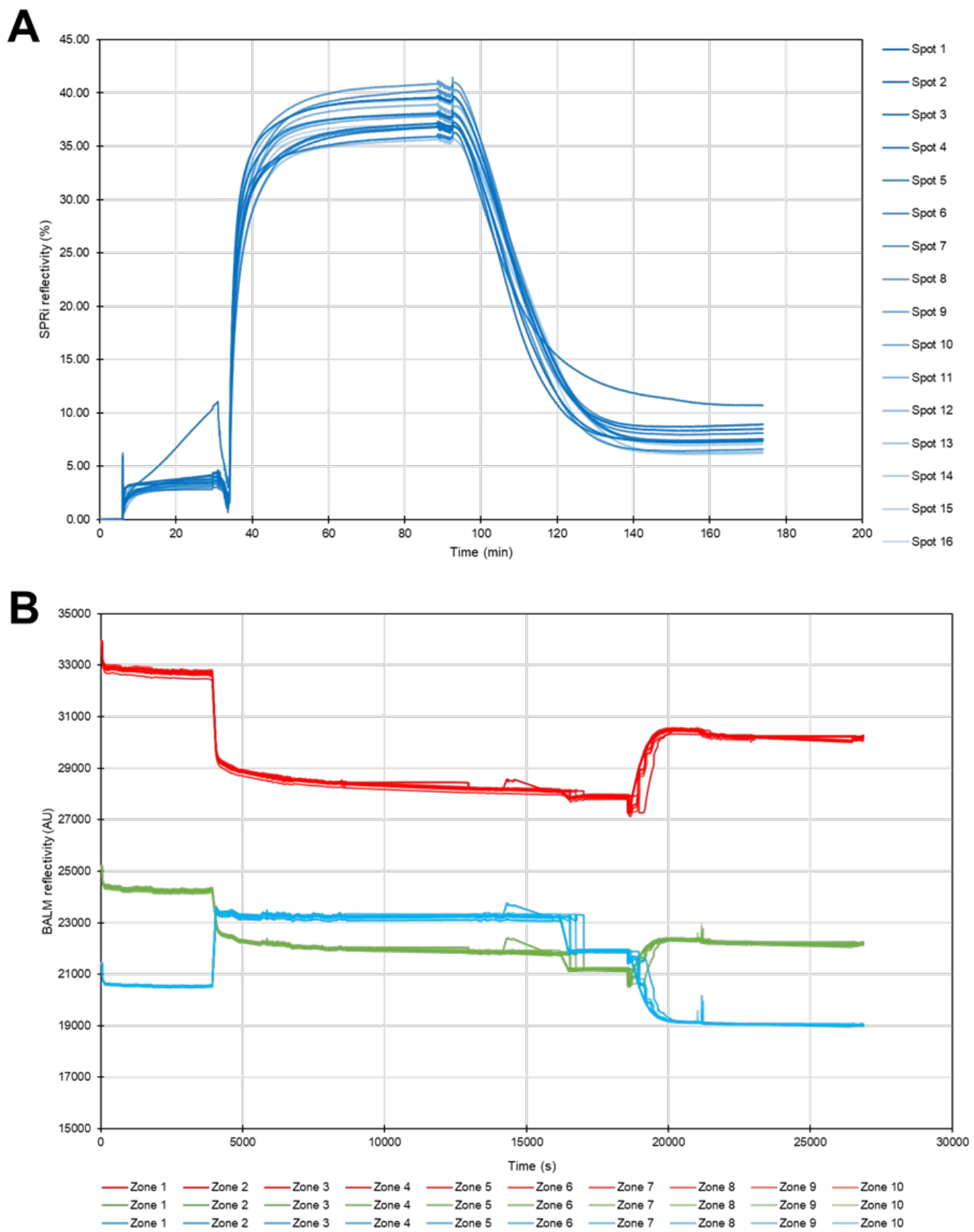

Finally, the linearity of BALM $\sqrt{R-R_{\min }}$ with $\Delta R$ (SPR) is excellent with the red channel and satisfactory with the green, as plotted in the figure below. It shows BALM $\sqrt{R-R_{\min }}$ as a function of $\Delta R$ (SPR) for the four saturation levels, i.e. for the initial buffer stage and the subsequent rinsing stages. The $R_{\min }$ used correspond to those previously optimized, i-.e. stage by stage, as explained in the first part of the Supplement 4. 


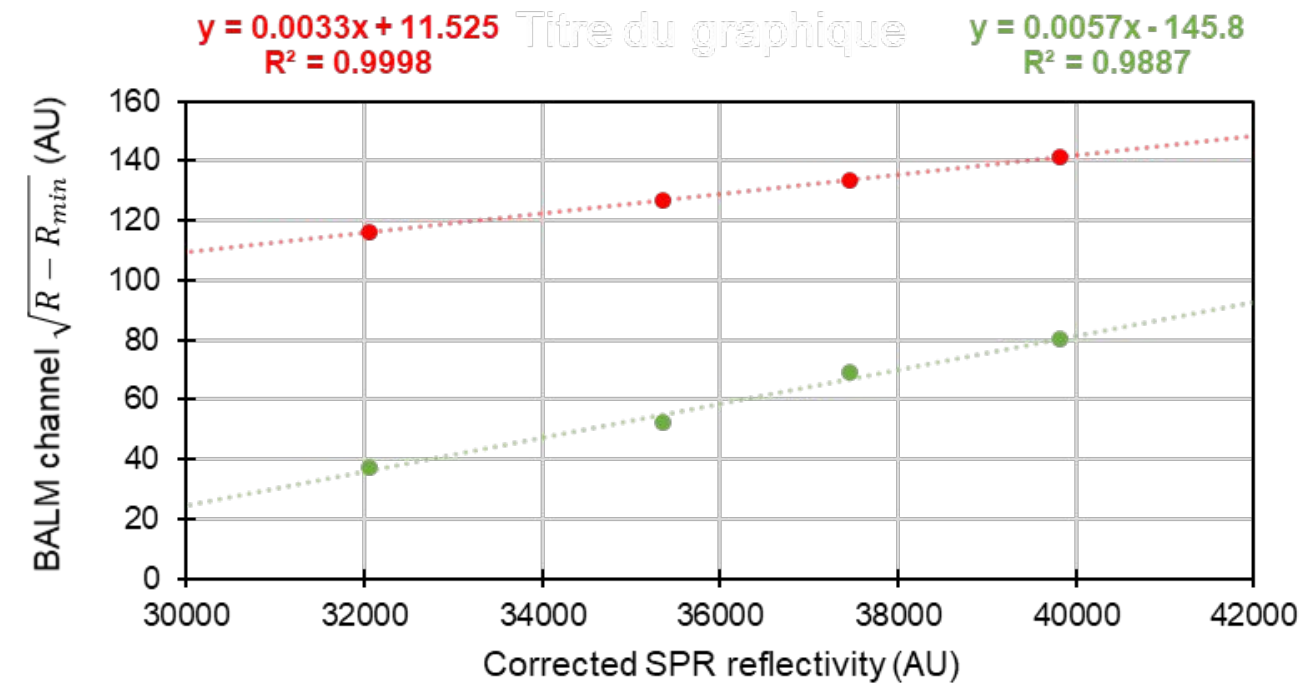




\section{Figure S6:}

Stage by stage SPR derivation of $\Delta R_{S P R}$ on the basis of Equation (1).

$\underline{\text { Starting buffer: }}$ refractive index $\equiv n_{0}$

$\Delta R_{S P R 0}=0$

Tether peptide grafting stage: refractive index of the current solute $\equiv n_{\mathrm{i}}$, volume fraction $\phi_{i}$.

$$
\Delta R_{S P R 1}=S\left\{\phi_{1}\left(n_{1}-n_{0}\right)+\left[n_{1}-n_{0}-\phi_{1}\left(n_{1}-n_{0}\right)\right] \alpha_{1} \frac{e_{1}}{\Lambda}\right\}
$$

The first term is the contribution of the solution, the so-called jump in index affecting the entire probing depth. Note that it is not affected by the reduction factor $\frac{e_{1}}{\Lambda}$, which explains its relative importance. The second term may be read $\left\{n_{1}-\left[n_{0}+\phi_{1}\left(n_{1}-n_{0}\right)\right]\right\} \alpha_{1} \frac{e_{1}}{\Lambda}$ showing that it conforms to Equation (1) after replacing $n_{0}$ by the new appropriate value. We finally get:

$\Delta R_{S P R 1}=S\left[\phi_{1}\left(n_{1}-n_{0}\right)+\left(1-\phi_{1}\right)\left(n_{1}-n_{0}\right) \alpha_{1} \frac{e_{1}}{\Lambda}\right]$

End of kinetics: The final (or saturation) value of $\alpha_{i} e_{i}$ is $\mathrm{A}_{i} E_{i}$, with $i$ the stage number.

$\Delta R_{\text {SPR } 1 \text { Sat }}=S\left[\phi_{1}\left(n_{1}-n_{0}\right)+\left(1-\phi_{1}\right)\left(n_{1}-n_{0}\right) \mathrm{A}_{1} \frac{E_{1}}{\Lambda}\right]$

After tether rinsing: ( $\triangle R_{S P R 2}$ is still counted with respect to the starting pure buffer)

$\Delta R_{S P R 2}=S\left(n_{1}-n_{0}\right) A_{1} \frac{E_{1}}{\Lambda}$

Liposome anchoring stage: liposome refractive index $\equiv n_{2}$

$$
\Delta R_{S P R 3}=S\left\{\phi_{2}\left(n_{2}-n_{0}\right)+\left[n_{1}-n_{0}-\phi_{2}\left(n_{2}-n_{0}\right)\right] \mathrm{A}_{1} \frac{E_{1}}{\Lambda}+\left[n_{2}-n_{0}-\phi_{2}\left(n_{2}-n_{0}\right)\right] \alpha_{2} \frac{e_{2}}{\Lambda}\right\}
$$

, where the first and second brackets may be read as before as, respectively, $n_{1}-\left[n_{0}+\phi_{2}\left(n_{2}-n_{0}\right)\right]$ and $n_{2}-\left[n_{0}+\phi_{2}\left(n_{2}-n_{0}\right)\right]$ to show the mechanism of the derivation.

$$
\begin{gathered}
\Delta R_{S P R 3}=S\left\{\phi_{2}\left(n_{2}-n_{0}\right)+\left[n_{1}-\phi_{2} n_{2}-\left(1-\phi_{2}\right) n_{0}\right] \mathrm{A}_{1} \frac{E_{1}}{\Lambda}+\left(1-\phi_{2}\right)\left(n_{2}-n_{0}\right) \alpha_{2} \frac{e_{2}}{\Lambda}\right\} \\
\Delta R_{S P R 3}=S\left\{\phi_{2}\left(n_{2}-n_{0}\right)+\left[n_{1}-n_{2}+\left(1-\phi_{2}\right)\left(n_{2}-n_{0}\right)\right] \mathrm{A}_{1} \frac{E_{1}}{\Lambda}+\left(1-\phi_{2}\right)\left(n_{2}-n_{0}\right) \alpha_{2} \frac{e_{2}}{\Lambda}\right\} \\
\Delta R_{S P R 3}=S\left[\phi_{2}\left(n_{2}-n_{0}\right)+\left(n_{1}-n_{2}\right) \mathrm{A}_{1} \frac{E_{1}}{\Lambda}+\left(1-\phi_{2}\right)\left(n_{2}-n_{0}\right)\left(\mathrm{A}_{1} \frac{E_{1}}{\Lambda}+\alpha_{2} \frac{e_{2}}{\Lambda}\right)\right] \quad \text { (S11-4) }
\end{gathered}
$$

The second term with $\left(n_{1}-n_{2}\right)$ in this expression may be viewed as a correction balancing the counting of $A_{1} \frac{E_{1}}{\Lambda}$ with the index $n_{2}$ in the third term.

End of kinetics:

$\Delta R_{S P R ~ 3 S a t}=S\left[\phi_{2}\left(n_{2}-n_{0}\right)+\left(n_{1}-n_{2}\right) \mathrm{A}_{1} \frac{E_{1}}{\Lambda}+\left(1-\phi_{2}\right)\left(n_{2}-n_{0}\right)\left(\mathrm{A}_{1} \frac{E_{1}}{\Lambda}+\mathrm{A}_{2} \frac{E_{2}}{\Lambda}\right)\right]$

After liposome rinsing: 


$$
\Delta R_{S P R 4}=S\left[\left(n_{1}-n_{0}\right) \mathrm{A}_{1} \frac{E_{1}}{\Lambda}+\left(n_{2}-n_{0}\right) \mathrm{A}_{2} \frac{E_{2}}{\Lambda}\right]
$$

Fusion stage: refractive index of the fusogenic peptide $\equiv n_{3}$

An additional difficulty appears with the lipid vanishing during the conversion of liposomes into bilayers. $A_{2} \frac{E_{2}}{\Lambda}$ is corrected by $-\alpha^{\prime}{ }_{2} \frac{e_{2}^{\prime}}{\Lambda}$ in presence of the $n_{3}$ solution. To account for this transformation, the liposomes are counted as an equivalent lipid layer with refractive index $n_{2}$.

$\Delta R_{S P R} 5=S$
$\left\{\phi_{3}\left(n_{3}-n_{0}\right)+\left(n_{1}-n_{3}\right) \mathrm{A}_{1} \frac{E_{1}}{\Lambda}+\left(n_{2}-n_{3}\right)\left(\mathrm{A}_{2} \frac{E_{2}}{\Lambda}-\alpha^{\prime}{ }_{2} \frac{e_{2}^{\prime}}{\Lambda}\right)+\left(1-\phi_{3}\right)\left(n_{3}-n_{0}\right)\left[\mathrm{A}_{1} \frac{E_{1}}{\Lambda}+\left(\mathrm{A}_{2} \frac{E_{2}}{\Lambda}-\alpha^{\prime}{ }_{2} \frac{e_{2}^{\prime}}{\Lambda}\right)+\alpha_{3} \frac{e_{3}}{\Lambda}\right]\right\}$

End of kinetics:

$\Delta R_{S P R} 5$ Sat $=S$

$\left\{\phi_{3}\left(n_{3}-n_{0}\right)+\left(n_{1}-n_{3}\right) \mathrm{A}_{1} \frac{E_{1}}{\Lambda}+\left(n_{2}-n_{3}\right)\left(\mathrm{A}_{2} \frac{E_{2}}{\Lambda}-\mathrm{A}_{2}^{\prime} \frac{E_{2}^{\prime}}{\Lambda}\right)+\left(1-\phi_{3}\right)\left(n_{3}-n_{0}\right)\left[\mathrm{A}_{1} \frac{E_{1}}{\Lambda}+\left(\mathrm{A}_{2} \frac{E_{2}}{\Lambda}-\mathrm{A}_{2}^{\prime} \frac{E_{2}^{\prime}}{\Lambda}\right)+\mathrm{A}_{3} \frac{E_{3}}{\Lambda}\right]\right\}$

After fusion rinsing:

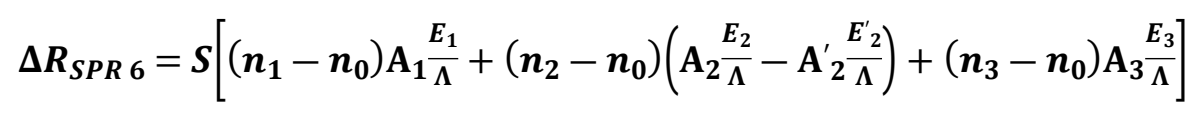

\section{Exploitation of the saturation levels:}

After rinsing levels are given by the blue equations (S11-0), (S11-3), (S11-6) and (S11-9). From the first to the last one, the contributions of the various deposited components add in sequence. The last term in (S11-9) with subscript 3 is relative to the fusogenic peptide. It is responsible for the brief bump at the beginning of the fusion stage, and then expected (from previous studies) to leave with the departing lipids. Hence $E_{3}=0$ and:

$\Delta R_{S P R \text { final }}=S\left[\left(n_{1}-n_{0}\right) \mathrm{A}_{1} \frac{E_{1}}{\Lambda}+\left(n_{2}-n_{0}\right)\left(\mathrm{A}_{2} \frac{E_{2}}{\Lambda}-\mathrm{A}_{2}^{\prime} \frac{E_{2}^{\prime}}{\Lambda}\right)\right]$

The after rinsing levels of the second and the third stage are both expressed in terms of lipid equivalent layers with refractive index $n_{2}$. Assuming a homogeneous bilayer at the end, (S11-10) can be written: $\Delta R_{S P R \text { final }}=S\left[\left(n_{1}-n_{0}\right) \mathrm{A}_{1} \frac{E_{1}}{\Lambda}+\left(n_{2}-n_{0}\right) \frac{h}{\Lambda}\right]$

, with $h$ the thickness of the bilayer.

\section{Conversion of the liposomes into equivalent bilayers:}

This conversion is based on the use of a liposome-equivalent lipid layer. In other words, the liposomes which are a mixture of lipids and water are described as the equivalent dense lipid layer, as if they were just emptied from water and shrunk to a dense state. This is supported as follows: 
i) the liposome refractive index $n_{L}$ is the average of lipid and buffer refractive indices $n_{2}$ and $n_{0}$ in their respective volume proportions in the lipsome $n_{L}=\left(\pi D^{2} h n_{2}+\frac{1}{6} \pi D^{3} n_{0}\right) /$ $\left(\pi D^{2} h+\frac{1}{6} \pi D^{3}\right)=\left(h n_{2}+\frac{D}{6} n_{0}\right) /\left(h+\frac{D}{6}\right) \cong n_{0}+6 \frac{h}{D}\left(n_{2}-n_{0}\right)$, with $D$ the liposome diameter.

ii) $\quad \Delta R_{S P R}$ with respect to the buffer reference is sensitive to the product $\left(n_{L}-n_{0}\right) D$ for the liposomes. At first order in $\frac{h}{D}$, we have $\left(n_{L}-n_{0}\right) D \cong 6 h\left(n_{2}-n_{0}\right)$, showing that the refractive index $n_{L}$ and thickness $\mathrm{D}$ of the liposome can be converted into the lipid refractive index $n_{2}$ and a lipid thickness $6 h$, which makes 6 equivalent bilayers per liposome footprint $\pi D^{2} / 4$.

iii) Then this equivalent thickness must be corrected by the number of liposomes footprints per surface unit, that is to say the liposome coverage ratio $A_{2}$. Its maximum possible value corresponds to the hexagonal packing density $A_{2} \cong 0.81$, leading to $\left(n_{L}-n_{0}\right) D \cong 4.84 h$ $\left(n_{2}-n_{0}\right)$. Another interesting reference is the random sphere adsorption density $A_{2}$ $\cong 0.64$, leading to $\left(n_{L}-n_{0}\right) D \cong 3.84 h\left(n_{2}-n_{0}\right)$.

\section{Liposome surface coverage:}

The after liposome after rinsing $\triangle R_{S P R} 4$ may now be written:

$\Delta R_{S P R 4}=S\left[\left(n_{1}-n_{0}\right) \mathrm{A}_{1} \frac{E_{1}}{\Lambda}+6 \mathrm{~A}_{2}\left(n_{2}-n_{0}\right) \frac{h}{\Lambda}\right]$

In addition to (S11-11) and (S11-12), the experiment gives:

$\Delta R_{S P R \text { final }}=0.4 \Delta R_{S P R} 4$

Combining the three equations, we get:

$$
-\Delta R_{S P R \text { final }}+\Delta R_{S P R 4}=0.6 \Delta R_{S P R 4}=5 \mathrm{~A}_{2}\left(n_{2}-n_{0}\right) \frac{h}{\Lambda}
$$

From where:

$$
\left(n_{1}-n_{0}\right) \mathrm{A}_{1} \frac{E_{1}}{\Lambda}=\frac{1.4}{0.6} \mathrm{~A}_{2}\left(n_{2}-n_{0}\right) \frac{h}{\Lambda}=2.33 \mathrm{~A}_{2}\left(n_{2}-n_{0}\right) \frac{h}{\Lambda}
$$

And:

$$
\Delta R_{S P R \text { final }}=0.4 S \Delta R_{S P R 4}=3.33 \mathrm{~A}_{2} S\left(n_{2}-n_{0}\right) \frac{h}{\Lambda}
$$

Applying afterwards the correction for the exponential decay (given to 1.59 in the main text and derived in Supplement 3), we finally get $(3.33 / 1.59=2,09)$ :

$\Delta R_{S P R \text { final }}=2.09 \mathrm{~A}_{2} S\left(n_{2}-n_{0}\right) \frac{h}{\Lambda}$

The above procedure may be used in general to get rid of some embarrassing contribution in the analysis of multistep SPR data.

$\mathrm{A}_{2}$ in this supplement corresponds to $\alpha$ in the text. 
Figure S7:

Local evolution of the sample at the submicron scale during the formation kinetics of the POPC peptBLM.

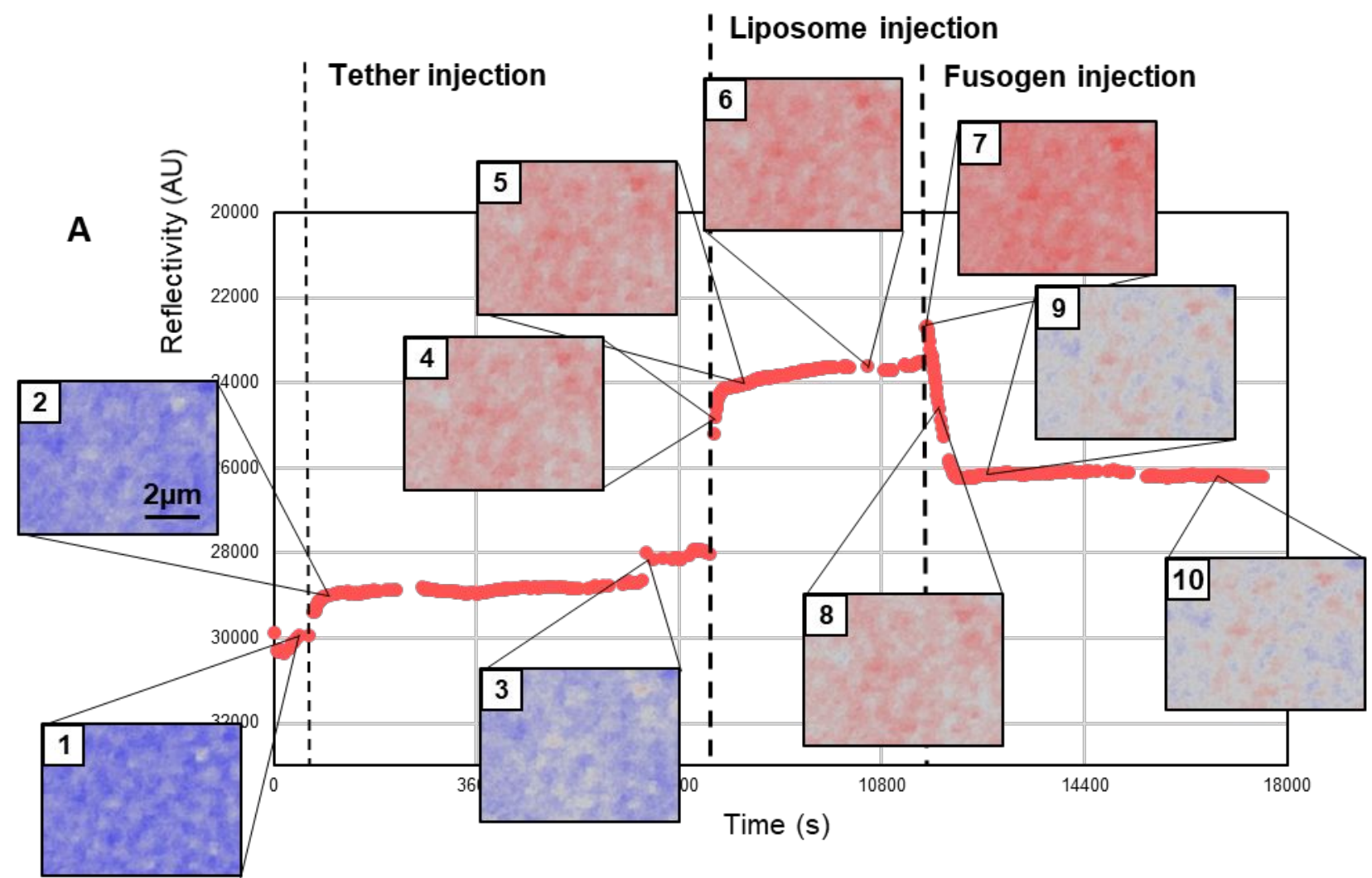

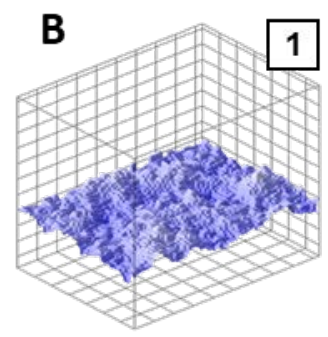

Buffer

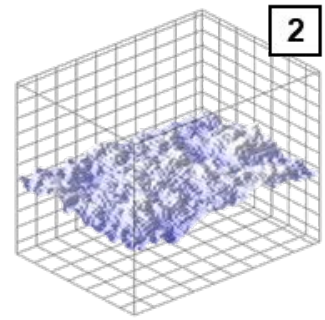

After tether peptide rinsing

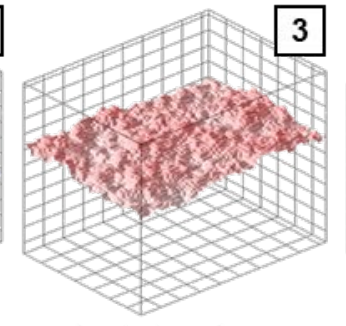

After liposome rinsing

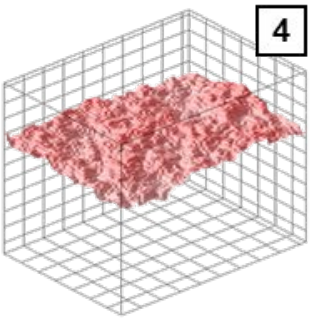

After Fusogenic peptide injection

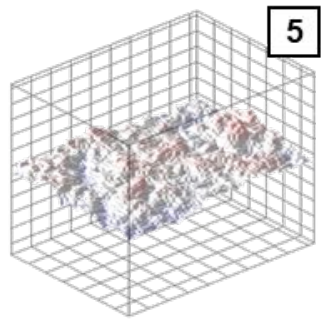

After fusogenic peptide rinsing

A. Series of high resolution images each associated with the corresponding point in the AntiBALM curve.

B. Surface plots displaying the local evolution of the surface patterns for significant steps in the formation process over a $0.9 \mu \mathrm{m} \times 1.2 \mu \mathrm{m}$ area.

The series of images show the same color shifts than obtained with the biomimetic liposomes: from an intense blue with the buffer to whiter hues after tether peptide injection; then red after the liposome injections and finally, a medium intensity after fusion. The intensity variation is easily followed with the surface plots in Supplement 6B, with a progressive swelling until liposome fusion. 
Figure S8:

Images used to make the Figure 3 plot profiles.
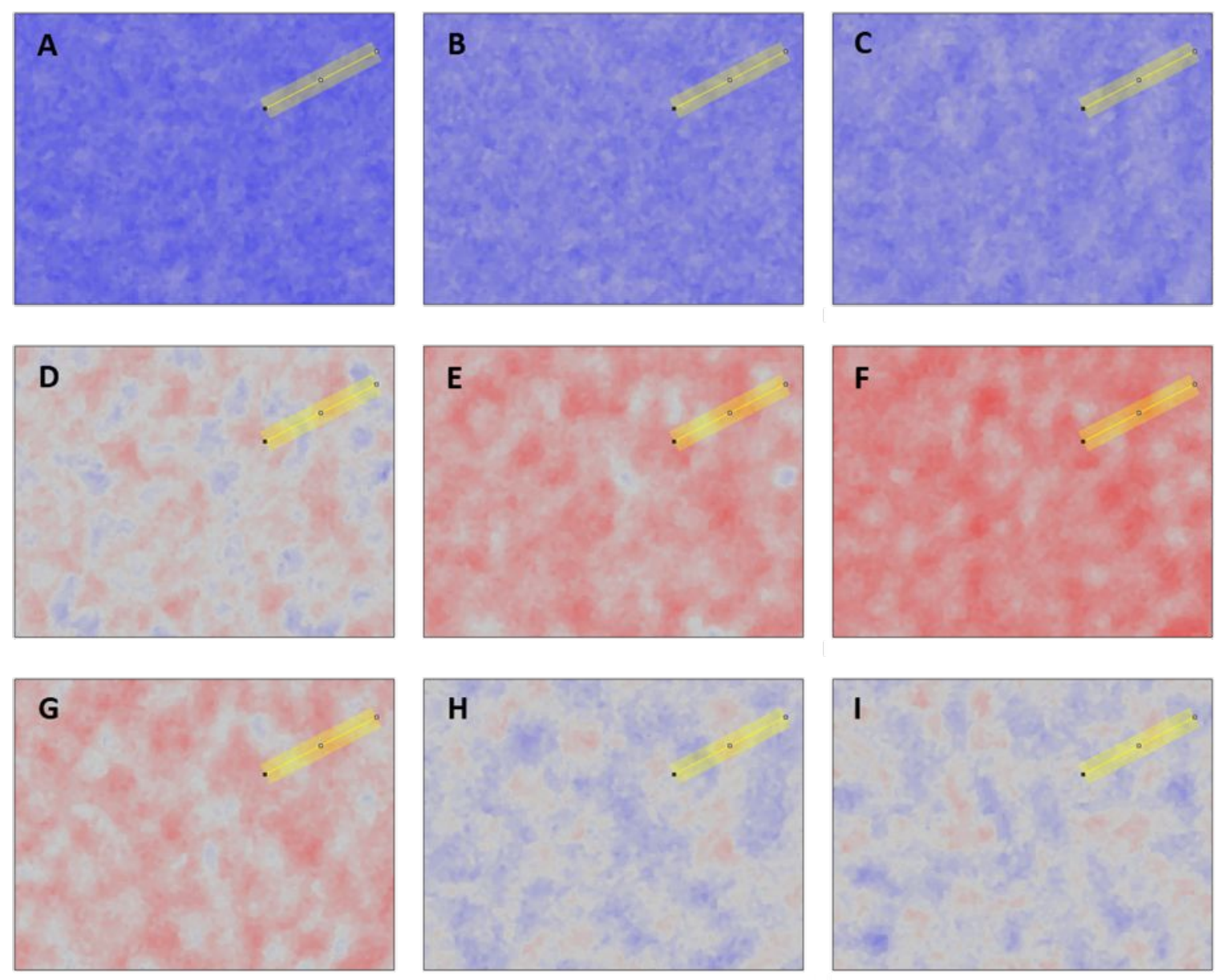

Each image was obtained after averaging the signal of four successive images, applying both an inverted phase LUT and a median 1-pixel wide filter. In yellow, the ten-pixel wide, $1.2 \mu \mathrm{m}$ long line used for the plot profiles. Each image is $3.1 \mu \mathrm{m}$ tall and $4.1 \mu \mathrm{m}$ wide. 


\section{Figure S9:}

Plot profiles along a ten-pixel wide, $1.2 \mu \mathrm{m}$ long line at nine significant steps of the peptide-tethered bilayer formation from POPC liposomes.
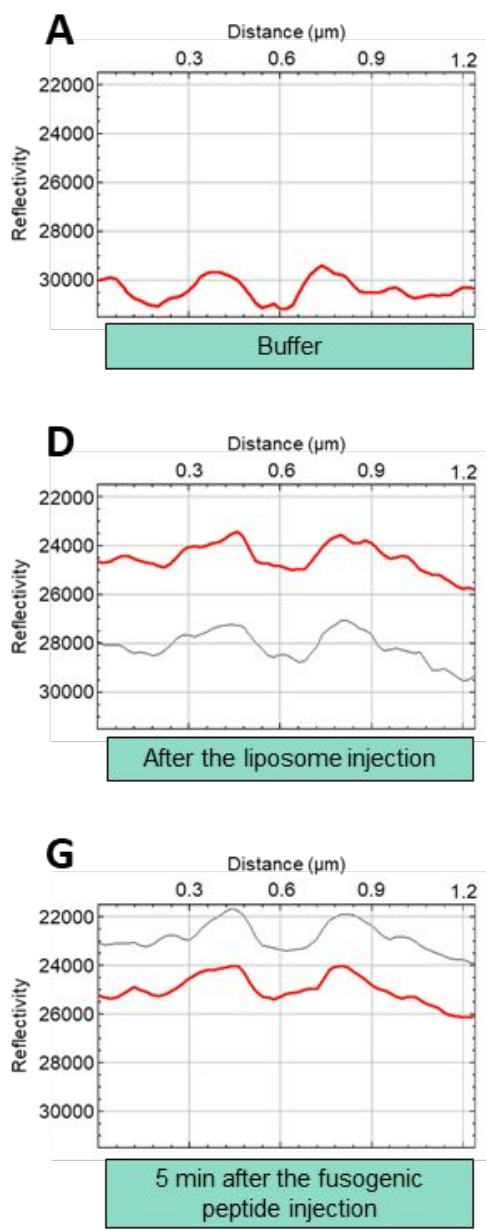
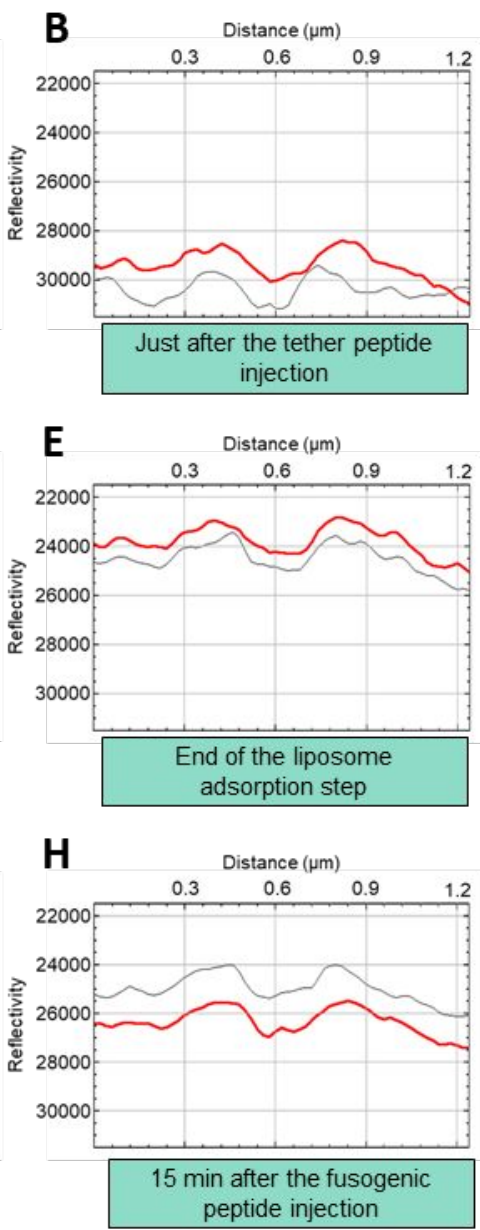
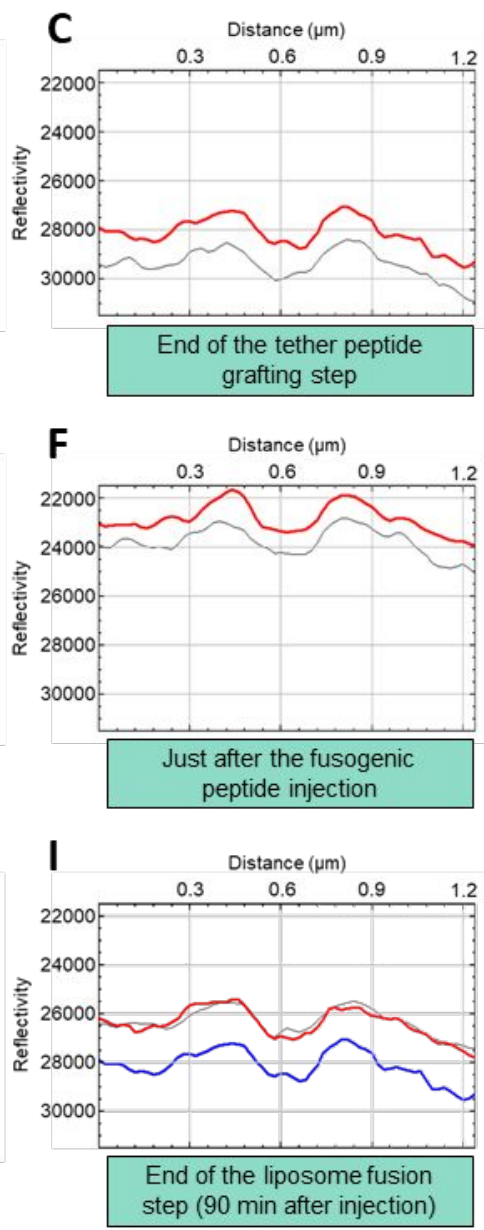

In red, the plot profile corresponding to the given step; in black the plot profile of the previous step; in blue (Supplement 8I) the plot profile corresponding to the end of the tether grafting step. Each plot profile was obtained after averaging the signal of four successive images, applying an inverted phase LUT and applying a median 1-pixel wide filter. The images used are available in Supplement 9.

The plot profiles obtained once again show the expected matter transfers in each step, with an elevation in the thickness during the tether peptide and the liposome injections, up to the early fusion step. Then, the thickness gradually decreases until a homogeneous layer is formed, as evidenced by the difference between the red (plot profile at the end of the fusion step) and blue (plot profile at the end of the tether peptide grafting step) curves in Supplement 81.

The plot profiles obtained with POPC liposomes are considerably more stable than the ones obtained with the biomimetic liposome composition. Such stark differences can be explained by the lipid segregation expected with the biomimetic mixture, as it is rich in both cholesterol and SM, which are lipids traditionally involved in segregation processes in biological membranes. Therefore, the liposome composition affects the bilayer formation mechanism. 


\section{Figure S10:}

Images used to make the Supplement 8 plot profiles.
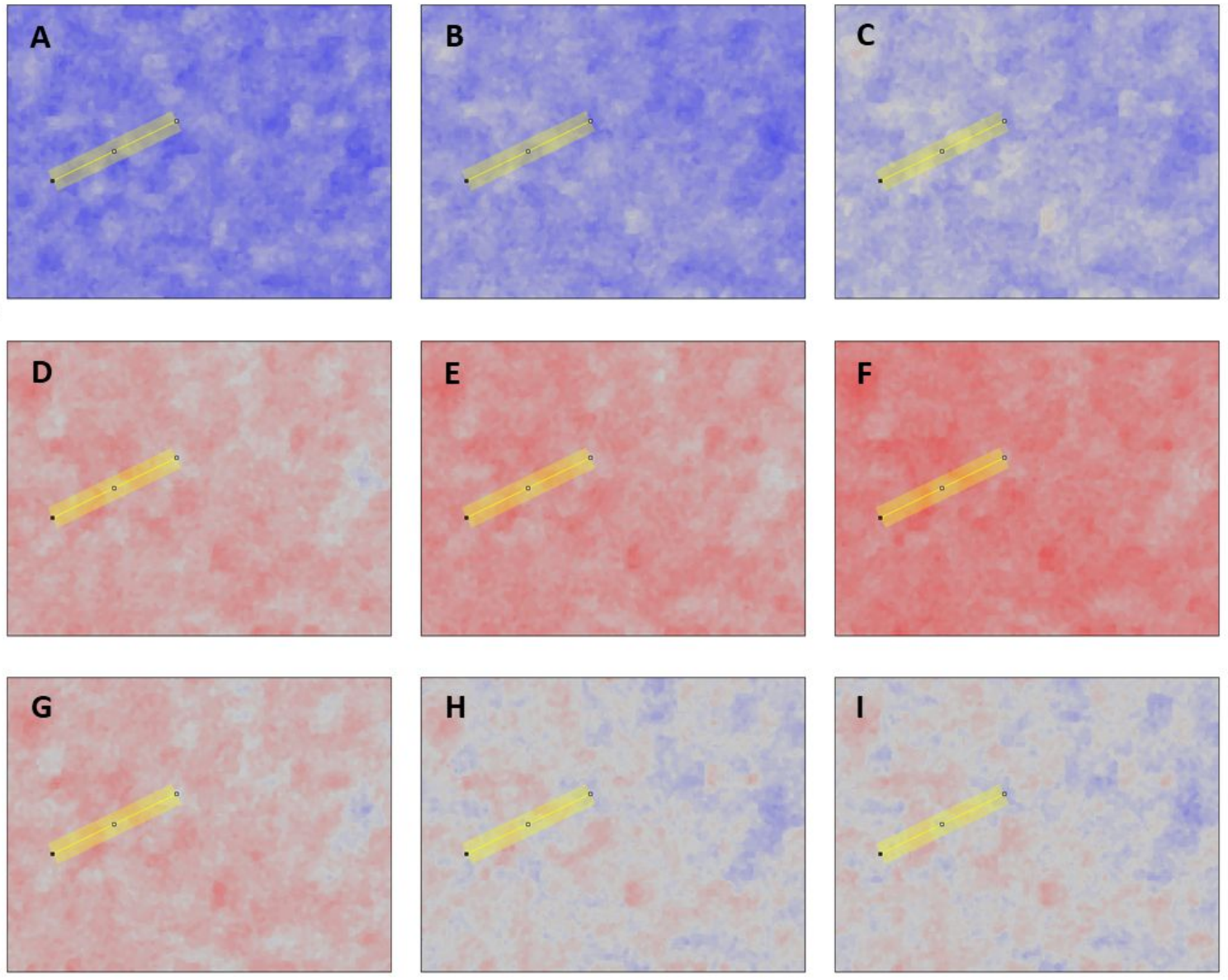

Each image was obtained after averaging the signal of four successive images, applying both an inverted phase LUT and a median, 1-pixel wide filter. In yellow, the ten-pixel wide, $1.2 \mu \mathrm{m}$ long line used for the plot profiles. Each image is $2.8 \mu \mathrm{m}$ tall and $3.7 \mu \mathrm{m}$ wide. 


\section{Figure S11:}

\section{Local kinetic measurements in the liposome stage.}

We work in the same $7 \times 5 \mu \mathrm{m}^{2}$ zone as used in Figure 2. Since the pattern appearing at the beginning is conserved for the whole duration of the kinetics, we could select two fixed spots in this zone corresponding to a high (A) and a low tether (B) density, respectively. These regions are drawn in the image below, corresponding to the end of the tether grafting step, where a median 1-pixel wide filter was applied and the contrast adjusted to maximize the height difference, in order to drive the eye toward the tether pattern on the surface. The zones areas are respectively $0,4 \mu \mathrm{m}^{2}(A)$ and $0,2 \mu \mathrm{m}^{2}$ (B).

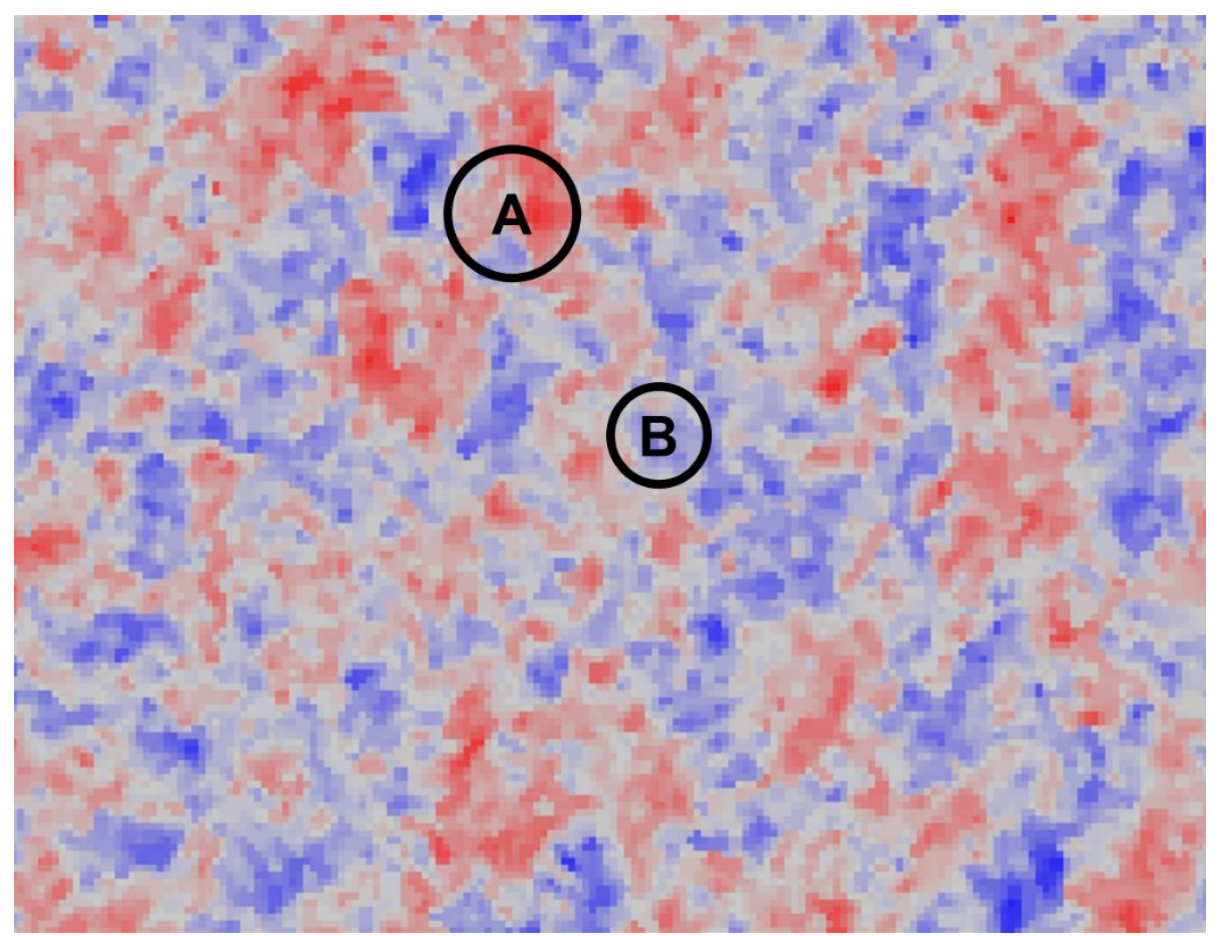

Then, we could follow the evolution of the BALM reflectivity in each defined zone. We choose the red channel because it is "almost" proportional to the film thickness. The results obtained in each stage are plotted under the image, with the high and the low tether density making the left and the right column. The y scale has the same amplitude in each pair. The quality of each curve would permit estimation of kinetic constants. This example demonstrates that BALM can provide local real time and in situ measurements of minute amounts much under the micron scale. 
High tether density
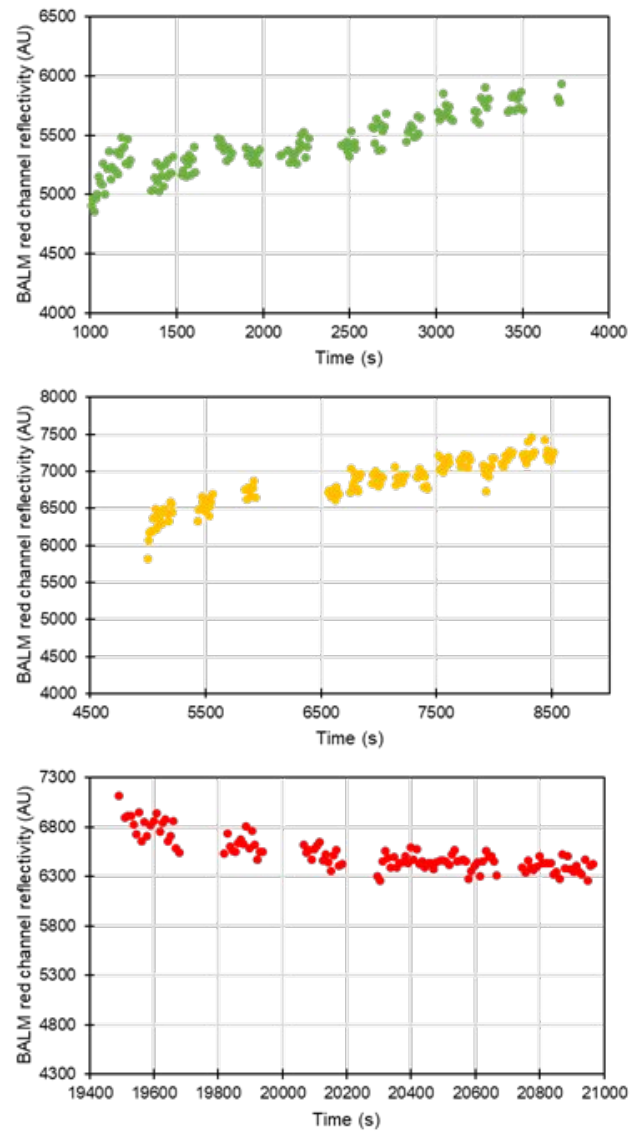

Low tether density

Tether peptide grafting stage
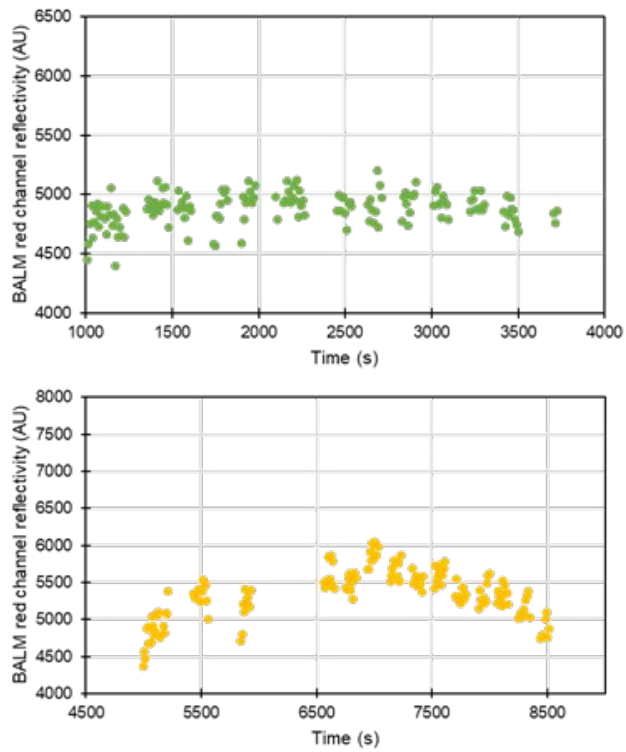

Fusion stage

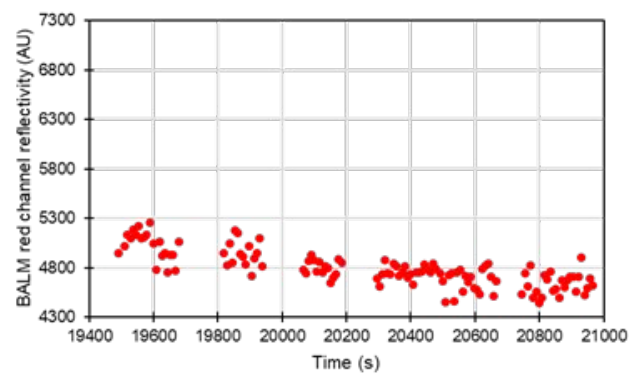

